\title{
Laser-Doppler Velocimetry Measurements Inside a Backward Curved Centrifugal Fan
}

\author{
TONG-MIIN LIOU ${ }^{\mathrm{a}, *}$ and MENG-YU CHEN ${ }^{\mathrm{b}}$ \\ a Professor, Department of Power Mechanical Engineering, National Tsing Hua University, Hsinchu, Taiwan, ROC; \\ ${ }^{\mathrm{b}}$ Graduate Student, Department of Power Mechanical Engineering, National Tsing Hua University
}

(Received 5 April 2000; In final form 27 May 2000)

\begin{abstract}
Laser-Doppler velocimetry (LDV) measurements are presented of relative mean velocity and turbulence intensity components inside the impeller passage of a centrifugal fan with twelve backward curved blades at design, under-design, and over-design flow rates. Additional LDV measurements were also performed at the volute outlet to examine the uniformity of the outlet flow for the three selected flow rates. Complementary flow visualization results in the tongue region are further presented. It is found that the number of characteristic flow regions and the average turbulence level increase with decreasing air flow rate. For the case of under-design flow rate, there are a through-flow region on the suction side, a reverse flow region on the pressure side, and a shear layer region in between. The corresponding average turbulence intensity is as high as $9.1 \%$ of blade tip velocity.
\end{abstract}

Keywords: Centrifugal fan; Tongue; Laser-Doppler velocimetry; Flow visualization

\section{INTRODUCTION}

Centrifugal fans (Fig. 1) are widely applied throughout industry, such as the ventilation system of tunnel, air conditioning system of building, cooling system of electronic devices, etc. The conventional method of evaluating the performance from the conditions at a fan's entrance and exit is insufficient. The improvements looked for, increased efficiency as well as reduced noise level, require an improved design method based on a better understanding of the fans' internal flow. However, measurements of flow in a centrifugal fan are difficult partly due to the complex geometry. Moreover, the flow passage is rotating and has curvature.

A large majority of previous research in centrifugal fans has focused on the impeller inlet and outlet flows not inside the impeller passage. Among them, Raj and Swim (1981) used a single hot wire probe to measure the mean velocities and the velocity fluctuations at the impeller exit of a small forward curved (FC) centrifugal fan. Their results showed the impeller flow to be highly

* Corresponding author. Tel.: 03 5719034, Fax: 035722840. 

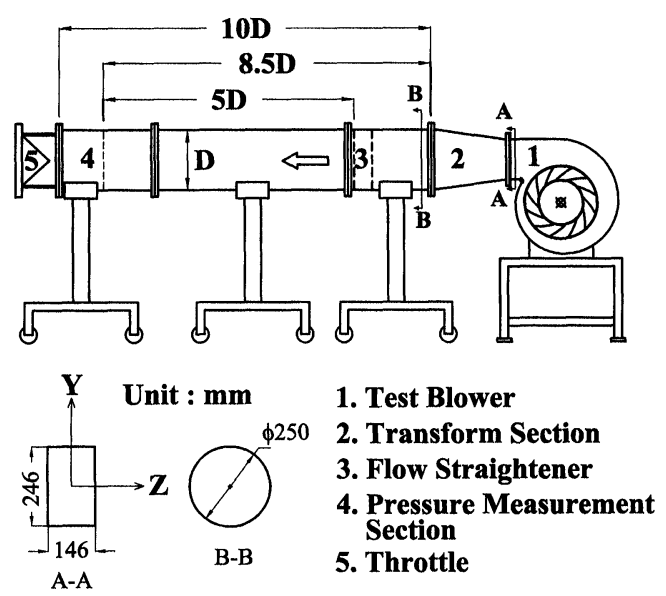

FIGURE 1 Schematic drawing of fan and outlet airway system.

turbulent and strongly three-dimensional (3-D). The inlet flow was found to fill only three quarters of the blade span. The shroud end of the impeller examined was thus an inactive or separated region. A jet-wake pattern occurred at the blade exit in the active flow region, as commonly observed at the exit of centrifugal compressor and pump impellers. Kind and Tobin (1990) measured the mean flow field at rotor inlet and rotor exit in three-squirrelcage fan configurations with a five-hole probe for flow rates below, near, and above the bestefficiency point (BEP). Their results showed that for large values of rotor exit-to-inlet area ratio of the fans investigated, flow separation of the incoming flow occurred and resulted in substantial axial and circumferential nonuniformity and reverse flow over much of the operating range, even at the BEP condition.

There appears to be very little published literature on internal flow in centrifugal fans. Nakayama et al. (1985) measured the distributions of the relative velocity in an impeller passage of a backward curved (BC) centrifugal fan using flow visualizations of spark tracing method and mist method. They observed the differences of flow patterns at three different flow rates. Surging phenomena were observed clearly at small flow rates. Kjork and Lofdahl (1989) measured the mean velocity components and Reynolds stresses in the blade passage of a centrifugal fan impeller employing a hot-wire probe rotating with the impeller. The fan has nine backward curved blades. All measurements were carried out at the design flow rate. Their results indicated an almost linear increase in the velocity profiles in the direction from the pressure side toward the suction side. However, a low velocity region is created near the suction side in the region closed to the shroud. They also found that the turbulent kinetic energy in the middle of the passage is almost constant in the flow direction.

It is clear that non-intrusive measurements in the blade passage are lacking due to the difficulties associated with optical access, seedings, unsteady nature, etc. Thus, the present study aims at performing Laser-Doppler velocimetry (LDV) measurements inside the impeller passage of a transparent centrifugal fan at design, over-design, and under-design flow rates. It is hoped that the gathered data can aid in a better understanding of flow characteristics inside the impeller passage at design and off-design conditions and provide a useful set of data base for numerical validations of CFD codes. Moreover, small variations in the flow field near the tongue may affect centrifugal fans' performance and loads markedly. In many cases manufacturers will adjust tongue shapes by trial and error in a fan prototype to improve the performance or to reduce noise. A preliminary examination through flow visualization on the fluid flow near the tongue is certainly valuable and, hence, is performed in the present work. Furthermore, the uniformity of the fluid flow downstream of the volute at various flow rates is an important index for evaluating the fan system and is therefore examined in the present study as well.

\section{APPARATUS AND EXPERIMENTAL CONDITIONS}

\section{Test Rig}

The test rig shown schematically in Figure 1 is designed according to AMCA (Air Movement and 
Control Association) 210-85 standard. It is composed of a commercially available centrifugal fan (TA SHENG, Model DT18E-7) to which an outlet side test airway is connected. At the inlet, the fan is fitted with a bellmouth which can be adjusted to obtain an appropriate inlet clearance of $2.5 \mathrm{~mm}$. The fan has an inlet diameter of $250 \mathrm{~mm}$ and an exit area of $146 \times 246 \mathrm{~mm}^{2}$. The width of the collector is 3.65 times the width of the impeller, and it has a spiral profile. There are twelve backward curved blades of $2 \mathrm{~mm}$ in thickness and $40 \mathrm{~mm}$ in height. Each blade is curved as an arc with a radius of $401 \mathrm{~mm}$ and arranged so that the center of the arc falls on a circle with a radius of $290 \mathrm{~mm}$ measured from the center of the impeller. The original steel case and impeller of the fan are replaced by the $8-\mathrm{mm}$ thick plexiglass having the same internal dimensions. The fan is driven by an electric motor of $2 \mathrm{HP}$ whose rotational speed is controlled by using a frequency converter. Its rectangular exit is connected to the AMCA $210-85$ test duct of $250 \mathrm{~mm}$ in diameter by a divergent transformation section. The test duct system consists of a straightener section, a pressure measuring system, and a throttling device of swivel damper type. The static and total pressure are measured by Pitot tubes as the mean value of the pressure at pressure measuring section, and the fan flow rate can thus be calculated in accordance with the AMCA 210-85 standard.

\section{LDV System}

The LDV experimental setup is similar to that described by Liou and Chen (1999). As shown in Figure 2, LDV optics is a two-color four beam two-component system. A 4-W argon-ion laser with $514.5 \mathrm{~nm}$ (green) and $488 \mathrm{~nm}$ (blue) lines provided the current light sources. Both forward and off-axis scattering configurations were used in the experiment; based on $1 / \mathrm{e}^{2}$ extent of light intensity, the former provided a probe volume of about $1.69 \mathrm{~mm}$ in length and $0.164 \mathrm{~mm}$ in diameter and the latter $0.74 \mathrm{~mm}$ in length and $0.164 \mathrm{~mm}$ in diameter inside the test section. The entire LDV system was mounted on a milling machine with

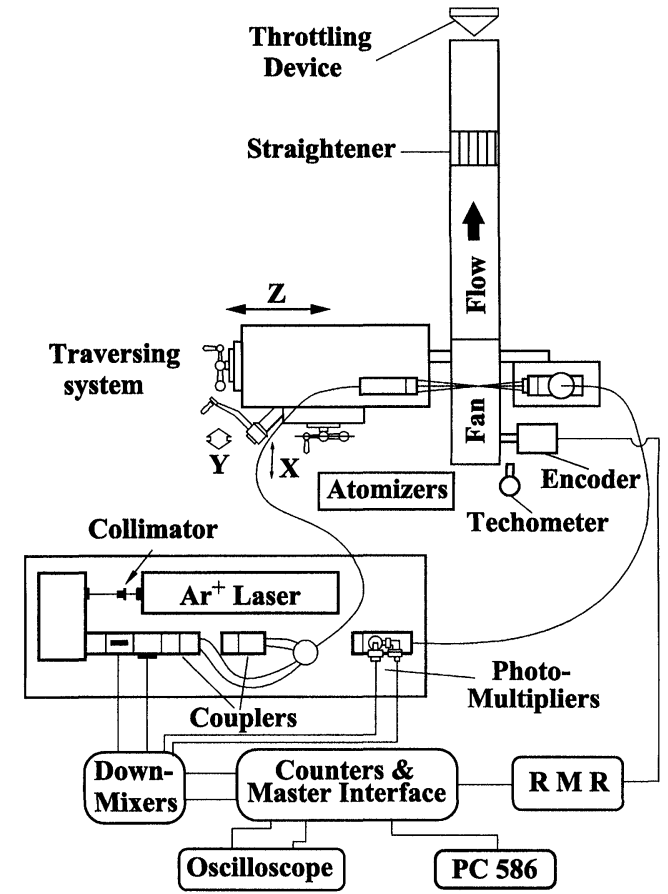

FIGURE 2 LDV experimental system.

four vibration-isolation mounts, allowing the probe volume to be positioned with $0.01 \mathrm{~mm}$ resolution. The light scattered from salt particles with a nominal $0.8 \mu \mathrm{m}$ size was collected into the photomultiplier and subsequently downmixed to the appropriate frequency shift of 0.1 to $10 \mathrm{MHz}$. Then two counter processors with 1 ns resolution were used to process the Doppler signals and feed the digital outputs into a PC-586 for storage and analysis. The phase angle in a given cycle was determined with an optical encoder attached to the pulse generator. A phase angle processor was used to generate a trigger pulse at any selectable phase angle. This trigger pulse was in turn input to an inhibit module which output a variable duration pulse to inhibit the counter processor over a selected phase angle interval. The positioning window of each measuring location was indexed by the rotary encoder with a resolution (ER) of 0.125 degree. When a velocity measurement was validated by the counter processor, the phase angle-velocity data was transferred to the PC-586 and recorded in the separate data bins. The 
circumferential length of a single data bin was from 0.54 to $0.71 \mathrm{~mm}$ as the measuring location varied from $r / R=0.76$ to $r / R=1.0$.

\section{Test Conditions}

The dimensions of the test airway and fan are depicted in Figures 1 and 3, respectively. Figure 3 also shows the configuration and coordinate system of the fan system. Figure 4 depicts the detailed measurement locations within the impeller passage. Flow field measurements were conducted within the impeller passage at over-design(143\%), design $(100 \%)$ and under-design( $48 \%)$ flow rates.

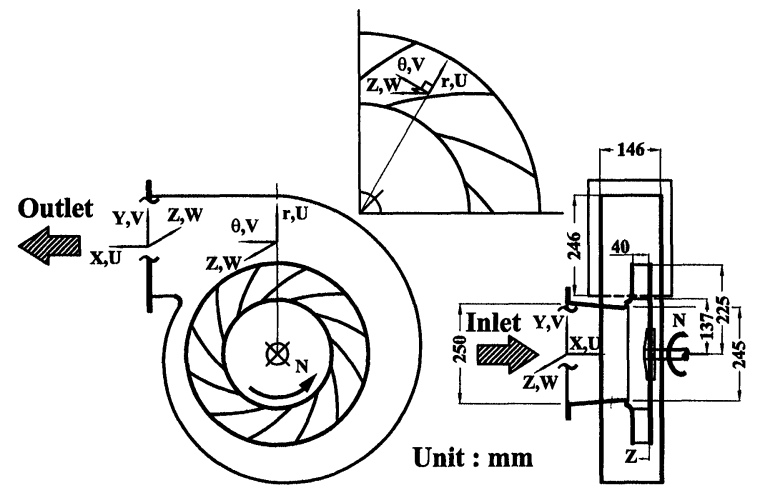

FIGURE 3 Sketch of configuration, coordinate system and dimensions of test fan.

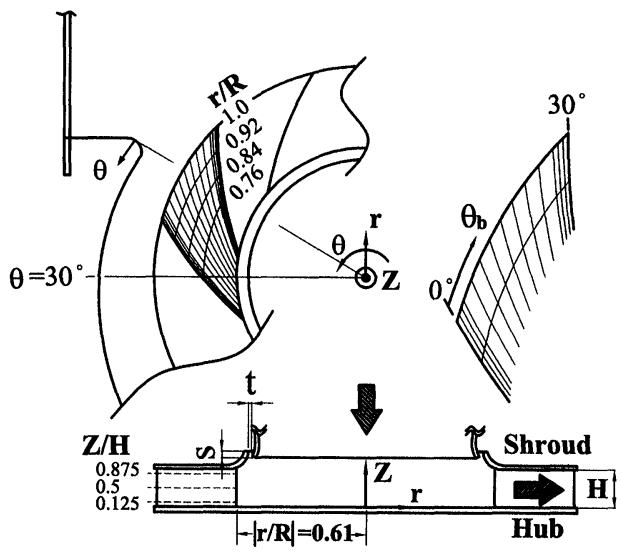

FIGURE 4 Sketch of coordinate system and measurement locations.
The Reynolds number based on the impeller diameter of $2 R=0.45 \mathrm{~m}$ and a fixed blade tip velocity of $U_{p}=41.23 \mathrm{~m} / \mathrm{s}$ (rotational speed of the impeller fixed at $1750 \mathrm{rpm}$ ) was $1.18 \times 10^{6}$. If $U_{p}$ is replaced by $U r_{b}$, the corresponding $R e$ are $2.18 \times 10^{5}, 1.52 \times 10^{5}$ and $0.73 \times 10^{5}$, respectively. The velocity measurements were made in $Z$ / $H=0.125,0.5$ and 0.875 planes between the impeller hub (or back plate) and shroud, as depicted in Figure 4. In each data plane the velocity measurements were performed along different radii $r / R=0.76,0.84,0.92$ and 1.0 and $\theta_{b}=1^{\circ}, 2^{\circ}, 3^{\circ}, 6^{\circ}, 9^{\circ}, 12^{\circ}, 15^{\circ}, 18^{\circ}, 21^{\circ}, 24^{\circ}, 27^{\circ}$, $28^{\circ}$ and $29^{\circ}$.

\section{Data Uncertainty}

The presented mean velocity and turbulence intensity components were calculated from the probability distribution function of the measurements. Typically $2000 \sim 4000$ realizations were ensemble averaged at each measuring location. Additional measurements of higher realizations (8000) in the high fluctuation regions were also taken to be sure of statistical errors in the ensemble averaged mean velocity and turbulence intensity were less than $0.018 U_{b}$ and $0.031 U_{b}$, respectively, for a 95 percent confidence level. Other sources of uncertainty were addressed in detail in the work of Liou and Chen (1999).

\section{RESULTS AND DISCUSSION}

\section{Velocity Vector Field}

Figure 5 depicts the measured relative mean velocity vector profiles, with respect to the coordinate system fixed at the impeller, in the impeller passage for the three cases investigated at a fan speed of $1750 \mathrm{rpm}$. Note that the mean velocities are made dimensionless with the blade tip velocity, $U_{p}$. It is seen that the flow on the pressure side is destabilized whereas the flow on the suction side is stabilized. This observation is 
in accordance with the theoretical investigations (Bradshaw, 1969) on the stability of boundarylayer flow and channel flow subject to rotation. In general, if the relative magnitude of the Coriolis force and the centrifugal force $\left(U r_{b} / \omega r \equiv\right.$ Rossby number $\equiv R o$, where $U r_{b}$ is the local bulk mean velocity across the cross section) is less than one,
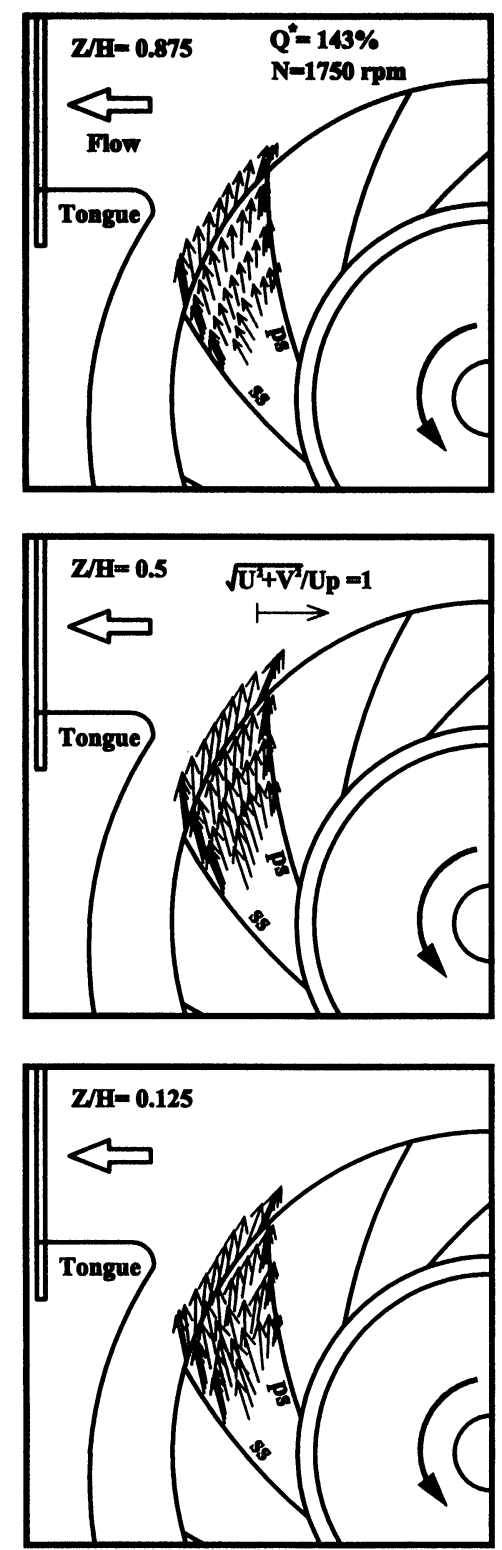

FIGURE 5a Vector profiles of relative mean velocity at high flow rate $\left(Q^{*}=143 \%\right)$ the stable location will be the suction side. If $R o$ exceeds one, then the stable location is the pressure side (Majidi and Siekmann, 1996). In the present study, $R o$ is in the range of 0.12 to 0.74 for halfimpeller radius $\leq r \leq$ impeller radius and $U r_{b}=$ $15.14 \mathrm{~m} / \mathrm{s} \quad\left(Q^{*}=143 \%\right), \quad 10.61 \mathrm{~m} / \mathrm{s} \quad\left(Q^{*}=100 \%\right)$, and $5.11 \mathrm{~m} / \mathrm{s}\left(Q^{*}=48 \%\right)$. Hence, the flow on the
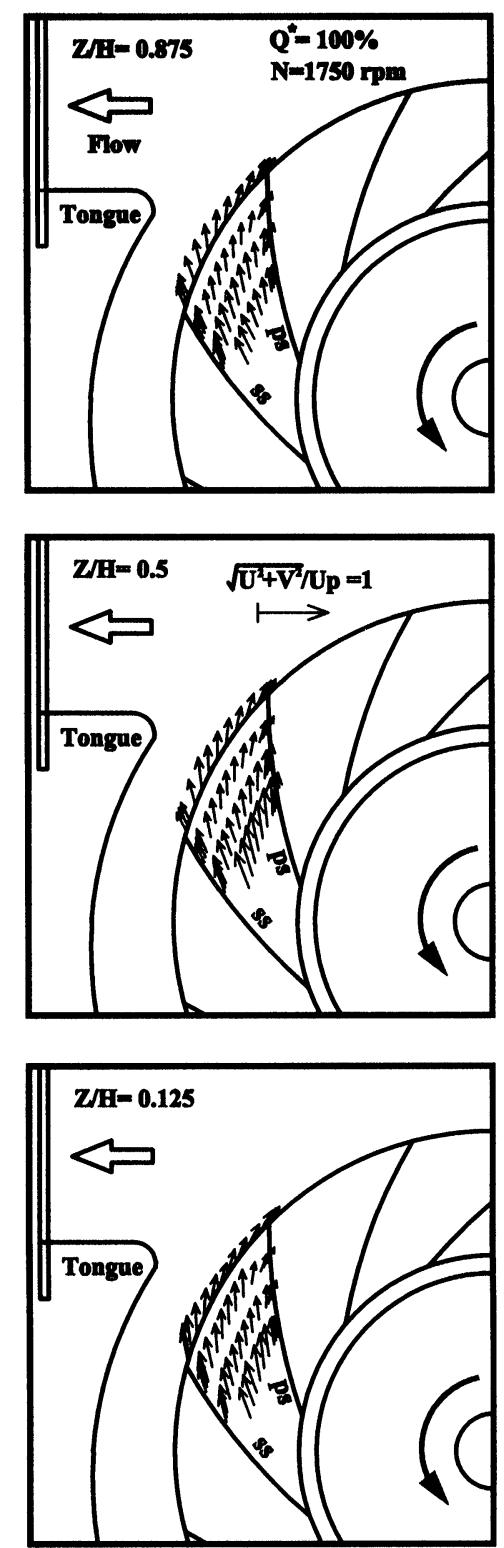

FIGURE $5 \mathrm{~b}$ Vector profiles of relative mean velocity at design flow rate $\left(Q^{*}=100 \%\right)$ 

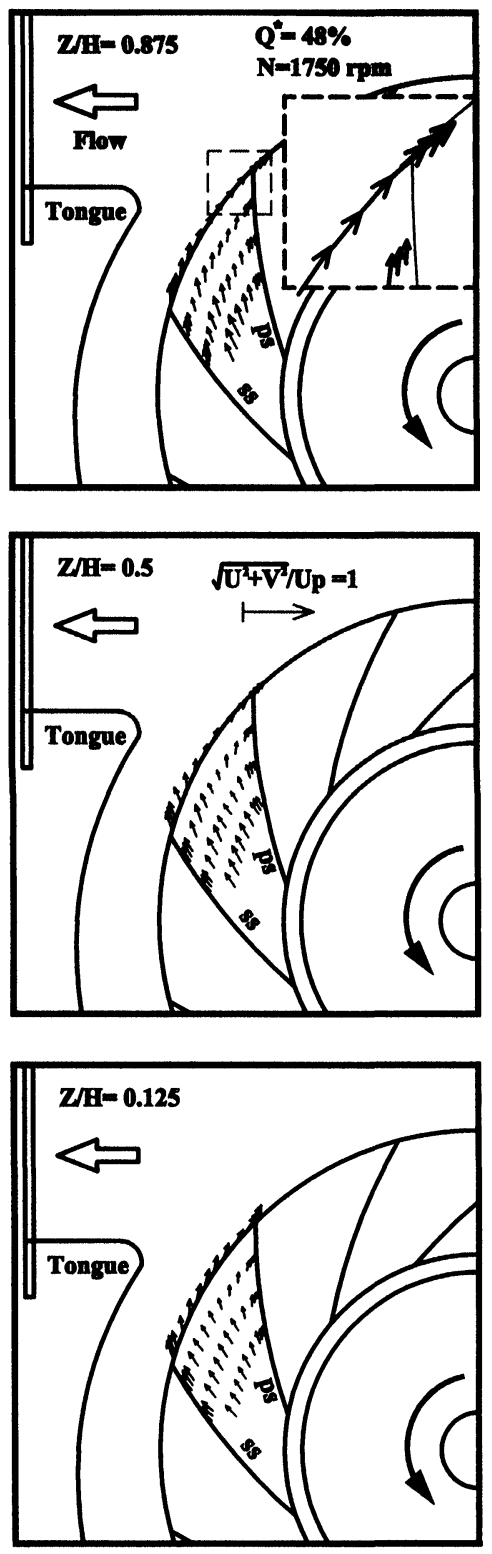

FIGURE 5c Vector profiles of relative mean velocity at low flow rate $\left(Q^{*}=48 \%\right)$

suction side is stabilized. The destabilization of the flow on the pressure side results in three characteristic regions: a through-flow region, a shear flow region, and a reverse flow region for the case of low flow rate $Q^{*}=48 \%$, as shown in Figure 6 which depicts the radial mean velocity and turbulence intensity profiles at impeller exit $(r / R=1)$
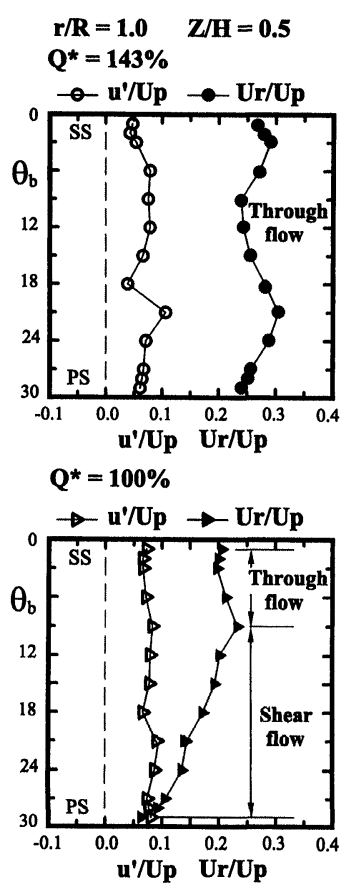

$Q^{*}=48 \%$

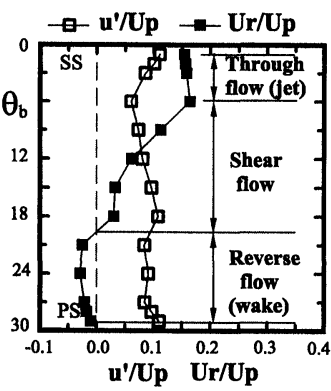

FIGURE 6 Reverse flow, shear flow, and through flow regions.

in the mid-height plane $(Z / H=0.5)$ of the impeller passage for the three $Q^{*}$ examined. For the case of design flow rate $Q^{*}=100 \%$, there is no reverse flow region. For the case of high flow rate $Q^{*}=143 \%$, there is only the through flow region. Hence, the flow rate $Q^{*}$ has marked influence on the flow characteristics in the impeller passage. Figure 6 also shows that the turbulence intensity profiles are rather uniform, a result similar to that reported by Kjork and Lofdahl (1989) using a hot wire probe. The average turbulence level increases with decreasing $Q^{*}$ or increasing number of characteristic regions. More specifically, the average 
$u^{\prime} / U_{p}$ levels are $6.6 \%, 7.6 \%$ and $9.1 \%$ of $U_{p}$ for $Q^{*}=143 \%, 100 \%$ and $48 \%$, respectively.

The evolution of the above-mentioned throughflow (or jet flow) for the three cases investigated can be better illustrated by using Figure 7 in terms of radial mean velocity distribution in the impeller passage for the case of $Q^{*}=143 \%$. The radial outward mean velocity is faster on the hub side than on the shroud side for most portions of the impeller passage due to the turning of the axial inflow into the radial direction. However, the nonuniformity of radial mean velocity profile becomes smaller at the design flow rate. It is well known that the boundary layer above an infinite rotating disk has a thickness of about $5.4 \sqrt{\nu / \omega}$ for the laminar flow case and $5.4 \sqrt{100 \nu / \omega}$ for the turbulent flow case (White, 1991). When nondimensionalized by the height of the impeller passage $H$, they are 0.04 and 0.4 , respectively. It is seen from Figure 7 that the jet-like portion moves toward the suction side as the flow proceeds from $r / R=0.76$ to $r / R=1.0$ the outlet edge of the impeller because of the imbalance between the Coriolis force and the pressure force. Within the boundary layer on the hub, the Coriolis force is smaller than the pressure force. The jet peak is therefore pushed toward the suction side.

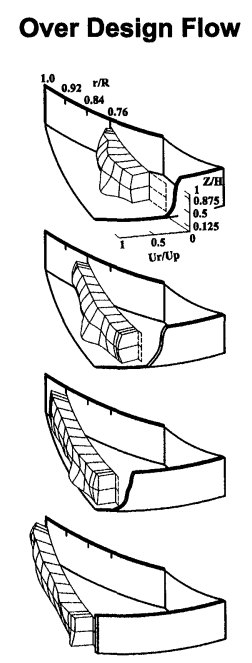

FIGURE 7 Evolution of radial mean velocity distribution in the impeller passage for $Q^{*}=143 \%$.

\section{Flow Visualization in the Tongue Region}

The fluid flow in the tongue region is split into two parts at tongue stagnation line. One part is directed toward the discharge side and the other re-enters the volute. It is interesting to know how the stagnation line changes with flow rate since the position of the stagnation line on the tongue is a useful characteristic parameter to be used for numerical validations. Flow visualizations using tufts are depicted in Figure 8. The results show that at high flow rates $\left(Q^{*}=143 \%\right)$ the stagnation line is on the inside of the tongue. At low flow rates $\left(Q^{*}=48 \%\right)$ the stagnation line moves into the discharge region.

\section{Flow Pattern at the Volute Outlet}

To reduce the irreversible mixing loss, the flow pattern needs to be uniform at the volute exit. Figure 9 depicts the mean velocity vector distributions in two orthogonal planes $Y_{o}=0$ and $Z_{o}=0$
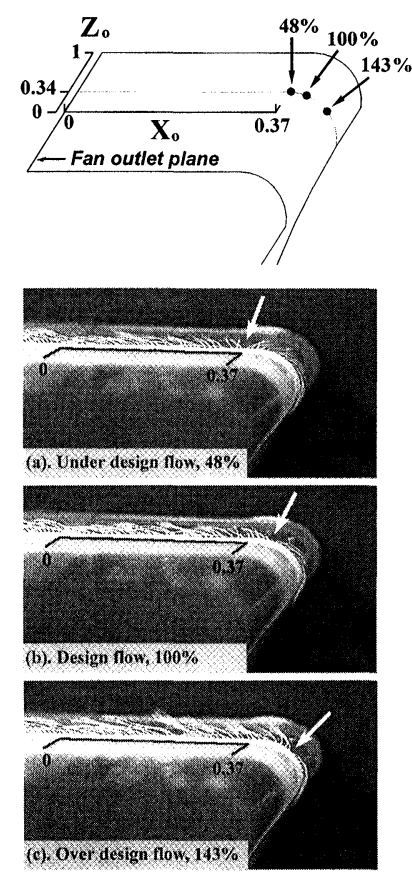

FIGURE 8 Tuft flow visualization in the tongue region for the three $Q^{*}$ examined (White arrow is used to identify the stagnation line). 

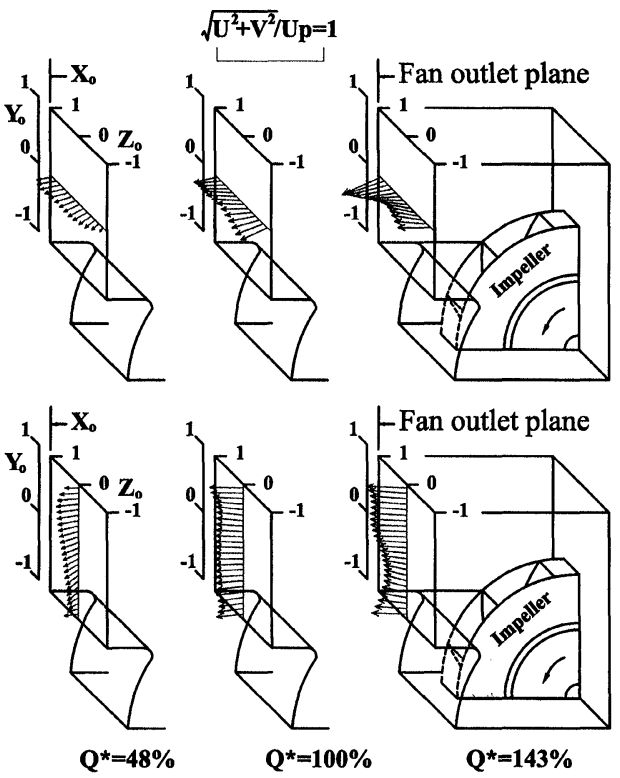

FIGURE 9 Mean velocity vector distributions in two orthogonal planes $Y_{o}=0$ and $Z_{o}=0$ for $Q^{*}=48 \%, 100 \%$ and $143 \%$.

for the three $Q^{*}$ studied. It is observed that a centrifugal fan operated at the design flow rate provides the most uniform mean velocity distributions at the volute outlet and, in turn, the minimum mixing loss among the three flow rates examined.

\section{CONCLUSIONS}

The following main results are drawn from the data presented:

1. The intra-passage fluid flow of the impeller is significantly affected by the air flow rate. For air flow rates below the design value, there exist three characteristic flow regions: a through-flow region on the suction side, a reverse flow region on the pressure side, and a shear layer region in between. At the design flow rate, the abovementioned flow reversal region disappears. For air flow rates beyond the design value, there exist only the through-flow region. The above variation of the characteristic flow regions with the air flow rate is believed to form a challenge to the computational fluid dynamics.
2. The average turbulence level is found to increase with increasing number of characteristic flow regions or with decreasing air flow rate.

3. At the design flow rate the stagnation line is at the tip of the tongue. For higher and lower (relative to the design flow rate) flow rates the stagnation line moves to the inside and the discharge side of the tongue, respectively. Such a pieces of information has not previously been available and can serve as a quantitative check to which results from computational fluid dynamic codes can be compared.

4. The volute outlet flow uniformity has been assessed for the three flow rates specified. It is found that the centrifugal fan investigated provides the most uniform outlet flow pattern if it is operated at the design flow rate.

\section{Acknowledgement}

Support for this work was provided by the National Science Council of the Republic of China under contact NSC86-2212-E007-087.

\section{NOMENCLATURE}

$D \quad$ exit pipe diameter $[=0.25 \mathrm{~m}]$

$h$ height of fan outlet cross section $[=0.246 \mathrm{~m}]$

$H \quad$ impeller blade height $[=0.04 \mathrm{~m}]$

$N \quad$ impeller rotation speed [ $=1750 \mathrm{rpm}$ ]

$Q \quad$ flow rate $\left[\mathrm{m}^{3} / \mathrm{min}\right]$

$Q_{d} \quad$ design flow rate $\left[=22 \mathrm{~m}^{3} / \mathrm{min}\right]$

$Q^{*} \quad$ dimensionless flow rate $\left[=Q / Q_{d}\right]$

$r \quad$ radius [m]

$r, \theta, Z \quad$ cylindrical coordinate system

$R \quad$ radius of impeller [ $=0.225 \mathrm{~m}$ ]

Re Reynolds number, $2 U R / \nu$

$s \quad$ axial clearance between bellmouth and impeller $[=0.005 \mathrm{~m}]$

radial clearance between bellmouth and impeller $[=0.0025 \mathrm{~m}]$ 


\begin{tabular}{|c|c|}
\hline$u, v, w$ & $\begin{array}{l}\text { fluctuating } \\
{[\mathrm{m} / \mathrm{s}]}\end{array}$ \\
\hline$u^{\prime}, v^{\prime}, w^{\prime}$ & $\begin{array}{l}\text { rms value of fluctuating velocity com- } \\
\text { ponents }\end{array}$ \\
\hline$U, V, W$ & mean velocity components $[\mathrm{m} / \mathrm{s}]$ \\
\hline$U_{b}$ & bulk mean velocity $[\mathrm{m} / \mathrm{s}]$ \\
\hline$U_{p}$ & blade tip velocity $[=41.23 \mathrm{~m} / \mathrm{s}]$ \\
\hline$U r$ & $\begin{array}{l}\text { radial relative velocity on impeller } \\
\text { passage }[\mathrm{m} / \mathrm{s}]\end{array}$ \\
\hline$U r_{b}$ & local bulk mean velocity $[\mathrm{m} / \mathrm{s}]$ \\
\hline$w$ & $\begin{array}{l}\text { width of fan outlet cross section } \\
{[=0.146 \mathrm{~m}]}\end{array}$ \\
\hline$X, Y, Z$ & rectangular coordinate system \\
\hline$X_{o}$ & $\begin{array}{l}\text { dimensionless coordinate of volute out- } \\
\text { let, } x(h+w) /(2 h w)\end{array}$ \\
\hline$Y_{o}$ & $\begin{array}{l}\text { dimensionless coordinate of volute out- } \\
\text { let, } 2 y / h\end{array}$ \\
\hline$Z_{o}$ & $\begin{array}{l}\text { dimensionless coordinate of volute out- } \\
\text { let, } 2 z / w\end{array}$ \\
\hline$\nu$ & $\begin{array}{l}\text { kinematic viscosity of the fluid } \\
{\left[=1.566 \times 10^{-5} \mathrm{~m}^{2} / \mathrm{s} \text { for air at } 23^{\circ} \mathrm{C}\right]}\end{array}$ \\
\hline$\rho$ & density $\left[\mathrm{kg} / \mathrm{m}^{3}\right]$ \\
\hline$\theta_{b}$ & $\begin{array}{l}\text { angular coordinate on the impeller } \\
\text { [degree] }\end{array}$ \\
\hline$\omega$ & impeller rotation speed $[\mathrm{rad} / \mathrm{s}]$ \\
\hline
\end{tabular}

\section{References}

Air Movement and Control Association, Inc. (1985) Publication 210-85, Laboratory Method of Testing Fans for Rating, American National Standard.

Bradshaw, P. (1969) The Analogy Between Streamline Curvature and Buoyancy in Turbulent Shear Flow, J. Fluid Mech., 36, $177-199$.

Brownell, R. B., Flack, R. D. and Kostrzewsky, G. J. (1985) Flow Visualization in the Tongue Region of a Centrifugal Pump, The Journal of Thermal Engineering, 4, 35-45.

Kind, R. J. and Tobin, M. G. (1990) Flow in a Centrifugal Fan of the Squirrel-Cage Type, ASME Journal of Turbomachinery, 112, 84-90.

Kjork, A. and Lofdahl, L. (1989) Hot Wire Measurements Inside a Centrifugal Fan Impeller, ASME Journal of Fluids Engineering, 111, 363-368.

Majidi, K. and Siekmann, H. E. (1996) Calculation of Secondary Flow in a Centrifugal Pump Using 3D Viscous Flow Computation Techniques, Proceedings of the 6th International Symposium on Transport Phenomena and Dynamics of Rotating Machinery, 2, 306-316.

Nakayama, Y., Yamamoto, T., Aoki, K. and Hiroaki, O. (1985) Measurement of Relative Velocity Distribution in Centrifugal Blower Impeller, Bulletin of JSME, 28(243), $1978-1985$.

Raj, D. and Swim, W. B. (1981) Measurements of the Mean Flow Velocity and Velocity Fluctuations at the Exit of an FC Centrifugal Fan Rotor, ASME Journal of Engineering for Power, 103, 393-399.

Liou, T. M. and Chen, C. C. (1999) LDV Study of Developing Flows Through a Smooth Duct with 180-Deg StraightCorner Turn, ASME Journal of Turbomachinery, 121, $167-174$.

White, F. M. (1991) Viscous Fluid Flow, McGraw-Hill, New York. 

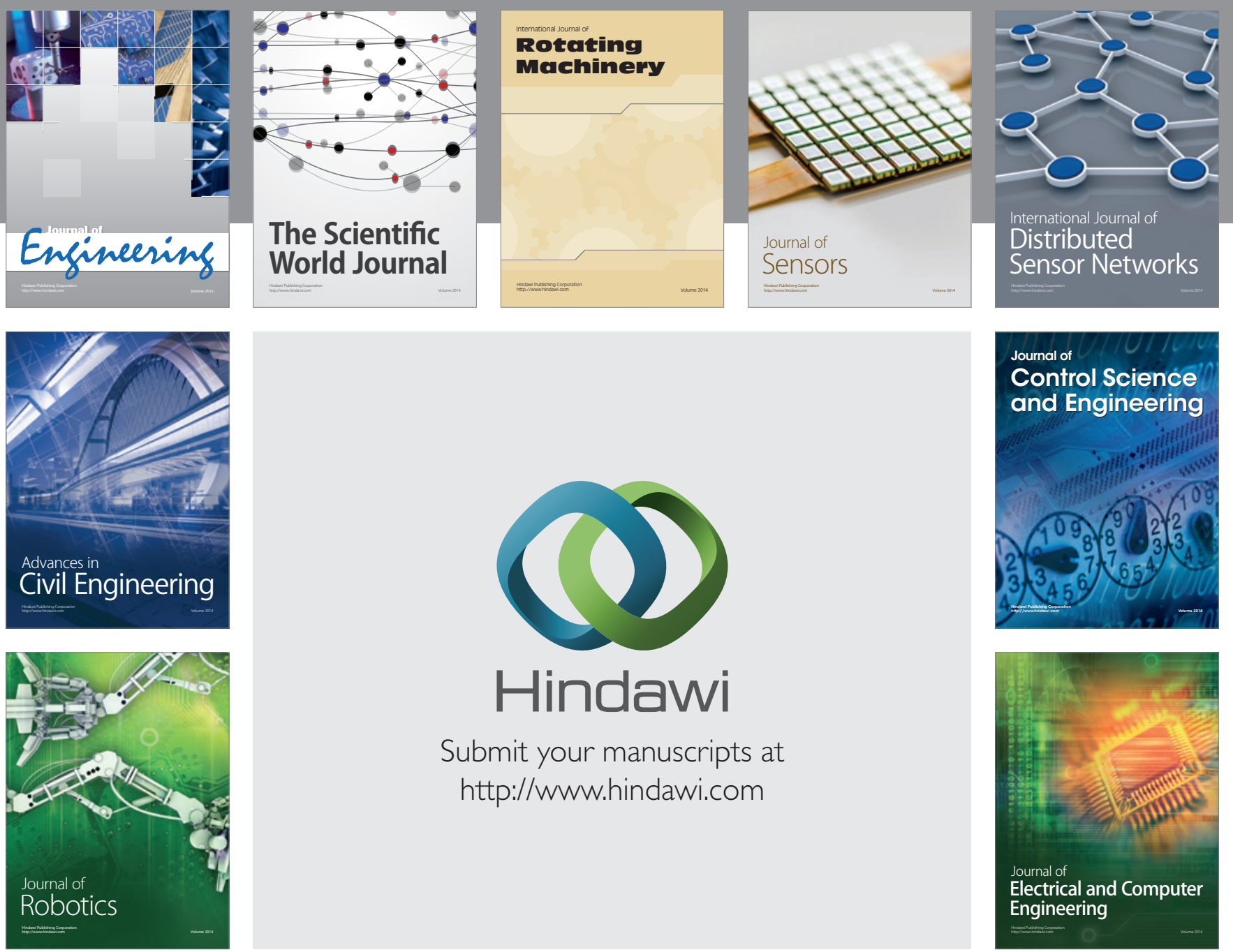

Submit your manuscripts at

http://www.hindawi.com
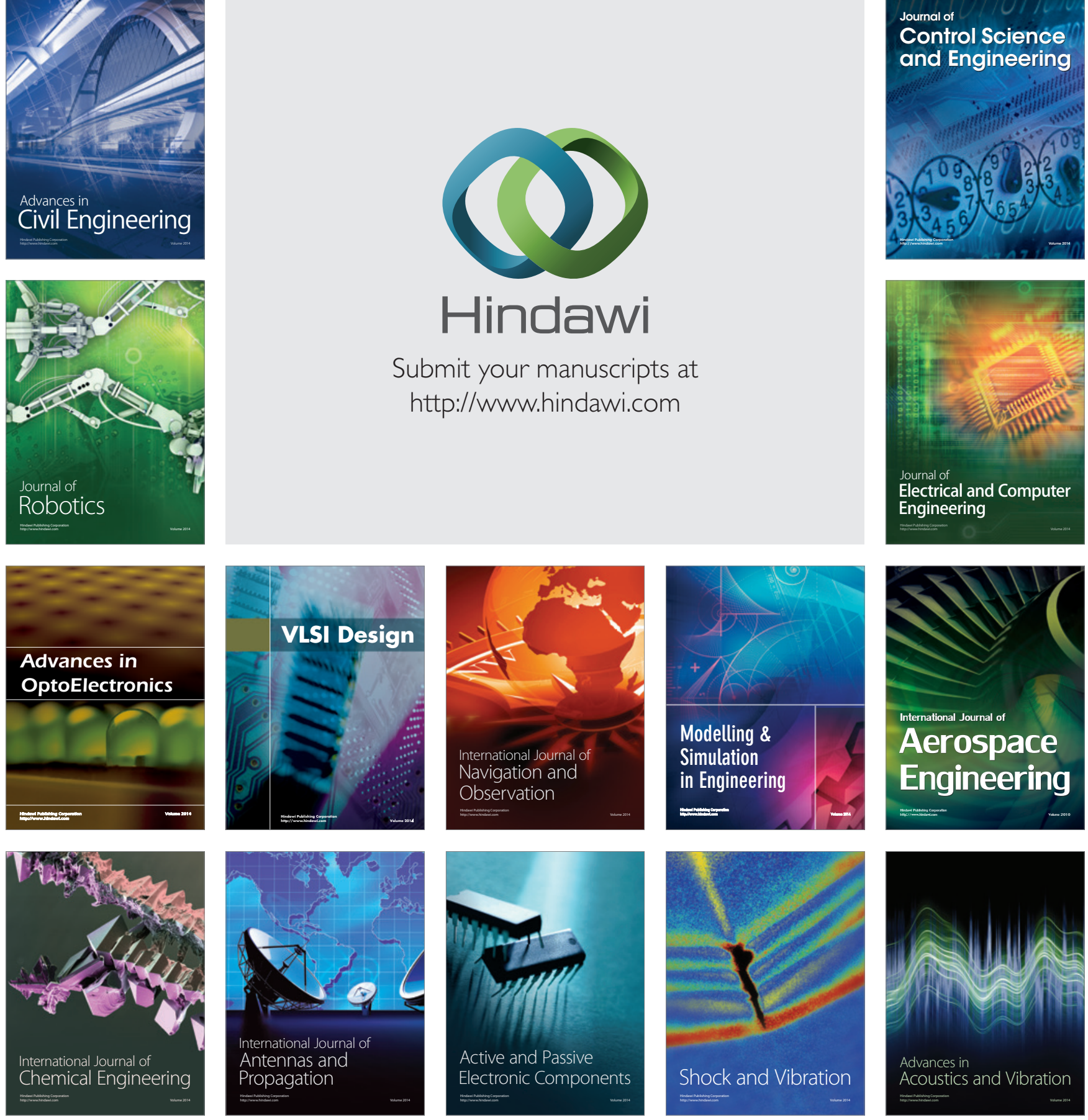